\title{
Avaliação da cor de resinas compostas através dos métodos visual e digital
}

Analysis of the color of composite resins through visual and digital methods

Evaluación del color de resinas compuestas a través de métodos visuales y digitales

Anderson da Silva SARMENTO' ${ }^{1}$

Hélio Gabriel Fernandes OLIVEIRA ${ }^{2}$

Kauana da Silva ANDRADE ${ }^{2}$

Fábio Victor Dias SILVA2

José Jhenikártery Maia de OLIVEIRA ${ }^{2}$

Andreia Medeiros Rodrigues CARDOSO ${ }^{3}$

Marcos Aurélio Vasconcelos LIMA JUNIOR ${ }^{4}$

Cirurgião Dentista pelo Centro Universitário de João Pessoa - UNIPÊ, 58053-000 João Pessoa-PB, Brasil

${ }^{2}$ Graduandos Curso de Graduação em Odontologia do Centro Universitário de João Pessoa - UNIPÊ, 58053-000 João Pessoa-PB, Brasil

${ }^{3}$ Professora Doutora do Curso de Graduação em Odontologia do Centro Universitário de João Pessoa - UNIPÉ, 58053-000 João Pessoa-PB, Brasil

${ }^{4}$ Professor Mestre do Curso de Graduação em Odontologia do Centro Universitário de João Pessoa - UNIPÊ, 58053-000 João Pessoa-PB, Brasil

\section{Resumo}

Objetivo: O presente estudo teve como objetivo verificar a correspondência de cor das resinas compostas em relação à Escala Vita, através do método visual e do método digital. Material e método: Trata-se de um estudo laboratorial in vitro realizado com 24 corpos de prova, utilizando resinas compostas de 8 diferentes marcas de resinas, nas cores $A 1$, A2 e A3, os quais foram submetidos à análise de tomada de cor, tanto pelo método visual quanto pelo método digital com auxílio de um scanner. Os dados foram analisados de forma descritiva e analítica por meio do Teste Qui-quadrado e o Teste de Fisher para comparar as frequências das coincidências pelo método visual e digital, de acordo com a marca, cor e método. Resultados: $41,67 \%$ das resinas apresentaram correspondência de cor quando avaliadas pelo método visual, enquanto o método digital apresentou $29,17 \%$ de correspondência de cor. As cores propostas não foram identificadas nem pelo método digital e nem pelo método visual (92.6\%), com diferença significativa $(p<0,05)$. Quando comparado as frequências de coincidências, houve mais coincidências pela identificação da cor pelo método visual do que pelo método digital $(p<0,05)$. Conclusão: Os métodos digital e visual clássico apresentaram pouca correspondência entre a cor proposta pelos fabricantes e a cor obtida após a polimerização das resinas. E quando comparadas as frequências de coincidências das cores, estas foram mais identificadas pelo método visual do que pelo método digital.

Descritores: Resinas Compostas; Cor; Testes Laboratoriais.

\section{Abstract}

Objective: The present study aimed to verify the color correspondence of composite resins in relation to the Vita Scale, through the visual method and the digital method. Material and method: This is an in vitro laboratory study carried out with 24 specimens, using resins composed of 8 different resin brands, in colors $A 1, A 2$ and $A 3$, which were subjected to the analysis of color taking, both by the visual method as well as by the digital method with the aid of a scanner. The data were analyzed in a descriptive and analytical way using the Chi-square test and the Fisher test to compare the frequencies of coincidences by the visual and digital method, according to the brand, color and method. Results: $41.67 \%$ of the resins showed color matching when evaluated by the visual method, while the digital method showed $29.17 \%$ color matching. The proposed colors were identified neither by the digital method nor by the visual method $(92.6 \%)$, with a significant difference $(p<0.05)$. When compared the frequencies of coincidences, there were more coincidences by identifying the color by the visual method than by the digital method $(p<0.05)$. Conclusion: The digital and classic visual methods showed little correspondence between the color proposed by the manufacturers and the color obtained after polymerization of resins. And when comparing the frequencies of color coincidences, they were more identified by the visual method than by the digital method.

Descriptors: Composite Resins; Color; Laboratory Test.

\section{Resumen}

Objetivo: El presente estudio tuvo como objetivo verificar la correspondencia de colores de las resinas compuestas en relación con la Escala Vita, utilizando el método visual y el método digital. Material y método: Se trata de un estudio de laboratorio in vitro realizado con 24 probetas, utilizando resinas compuestas por 8 marcas de resina diferentes, en los colores A1, A2 y A3, las cuales fueron sometidas al análisis de toma de color, tanto por el método visual como así como por el método digital con la ayuda de un escáner. Los datos se analizaron de forma descriptiva y analítica mediante la prueba de Chi-cuadrado y la prueba de Fisher para comparar las frecuencias de coincidencias por el método visual y digital, según marca, color y método. Resultados: el $41,67 \%$ de las resinas mostraron coincidencia de color cuando se evaluaron por el método visual, mientras que el método digital mostró una coincidencia de color del $29,17 \%$. Los colores propuestos no fueron identificados ni por el método digital ni por el método visual $(92,6 \%)$, con una diferencia significativa $(p<0,05)$. Al comparar las frecuencias de coincidencias, hubo más coincidencias al identificar el color por el método visual que por el método digital $(p<0.05)$. Conclusión: Los métodos visuales digitales y clásicos mostraron poca correspondencia entre el color propuesto por los fabricantes y el color obtenido tras la polimerización de resinas. Y al comparar las frecuencias de las coincidencias de color, se identificaron más por el método visual que por el método digital.

Descriptores: Resinas Compuestas; Color; Prueba de Laboratorio.

INTRODUÇÃO

\section{A procura por procedimentos estéticos é} cada vez mais frequente nos consultórios odontológicos, devido à crescente preocupação da sociedade de enquadrar-se nos padrões sociais estabelecidos. Baseado nisso, 0 tratamento restaurador, seja ele direto ou indireto, não deve focar apenas na reabilitação funcional dos dentes, mas também, na capacidade de restabelecer um sorriso que atenda às necessidades e às expectativas do paciente, fornecendo um resultado duradouro e com o aspecto mais natural possível ${ }^{1,2}$.

$$
\text { A seleção de cor é uma etapa }
$$
extremamente importante para o sucesso da 
terapia restauradora, pois a cor é um dos principais parâmetros utilizados pelos pacientes para mensurar a qualidade do procedimento restaurador. A construção de uma restauração com o aspecto mais natural possível é um dos maiores desafios para a odontologia restauradora, já que uma das principais causas da insatisfação com os tratamentos restauradores estéticos é a diferença de cor entre dente e restauração ${ }^{3-6}$.

$\mathrm{Na}$ Odontologia, os principais métodos utilizados para seleção da cor são o método visual e o instrumental. O método visual referese à seleção visual das cores através do olho humano, no entanto, esse método pode ser considerado como subjetivo, pois os indivíduos podem ter diferentes interpretações. $E$ os resultados esperados podem ser inconsistentes e insatisfatórios devido à complexidade das cores disponíveis e da influência de fatores externos, como a iluminação e temperatura do ambiente, a maquiagem $\mathrm{e}$ as roupas do paciente $^{7,8}$.

O método instrumental ou digital baseiase em instrumentos desenvolvidos para superar as limitações do método visual, como, por exemplo, os scanners intraorais. Ao comparar o scanner intraoral 3Shape Trios com o método visual, observou-se que o scanner intraoral pode ser utilizado como uma alternativa para seleção da cor ${ }^{9}$.

A confiabilidade na identificação dos tons dos dentes promove a redução no tempo clínico, nas etapas no fluxo de trabalho e adiciona consistência e precisão ao procedimento. Entretanto, ainda não parece claro qual método é o mais confiável, o visual ou o digital, o que tem feito com que muitos profissionais ainda elejam o método visual, mesmo com acesso à tecnologia ${ }^{5,6}$.

Diante do exposto, este estudo teve como objetivo verificar a efetividade da correspondência de cor entre diversas marcas de resinas compostas, utilizando o método visual e o método digital, a fim de identificar quais métodos asseguram a melhor correspondência entre as cores propostas e as cores obtidas após a polimerização.

\section{MATERIAL E MÉTODO}

Trata-se de um estudo laboratorial in vitro, realizado no laboratório de habilidades do Curso de Bacharelado em Odontologia do Centro Universitário de João Pessoa (UNIPÊ), onde foram desenvolvidos os corpos de prova, e no Centro de Diagnóstico Medical Face, onde a aferição das cores foi realizada com o Scanner Intraoral Trios3®, ambos localizados na cidade de João Pessoa - PB, Brasil. A fase experimental foi desenvolvida em novembro de 2020. A pesquisa foi realizada com 24 corpos de provas, utilizando resinas compostas microhíbridas, nanohíbridas, híbrida e microparticulada, nas cores $A 1, A 2$ e $A 3$, de diferentes marcas comerciais, produzidas por diferentes fabricantes e em diversos países (Figura 1 e Quadro 1), e que foram submetidos à análise de cor através do método visual clássico e por meio digital com auxílio de um scanner.

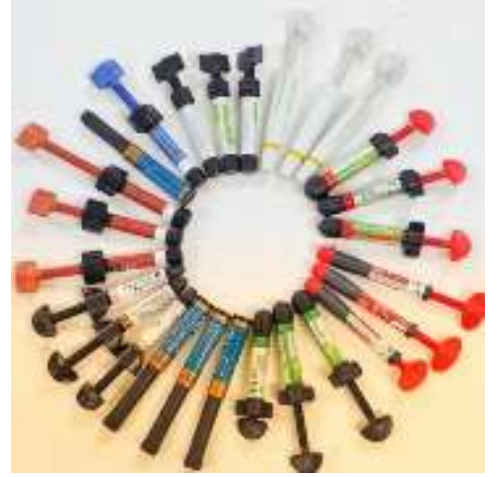

Figura 1: Resinas compostas utilizadas na pesquisa (Fonte: Dados da pesquisa, 2020).

Quadro 1 - Características das resinas compostas utilizadas na pesquisa.

\begin{tabular}{|l|l|l|}
\hline Nome comercial & Fabricante & Partículas \\
\hline Filtek Z10o & 3MEspe/ St. Paul, EUA & Microhíbrida \\
\hline Fill MagicG & $\begin{array}{l}\text { Coltene - Vigodent/ Rio de Janeiro, } \\
\text { RJ, Brasil }\end{array}$ & Microhíbrida \\
\hline Charisma Classic & $\begin{array}{l}\text { Kulzer/Mitsui Chemicals Group Inc } \\
\text { Tóquio, Japão }\end{array}$ & Microhíbrida \\
\hline LLiss & FGM/ Joinville, SC, Brasil & Nanohíbrida \\
\hline Spectra Smart & $\begin{array}{l}\text { Dentsply Sirona } \\
\text { Pirassununga, SP, Brasil }\end{array}$ & Nanohíbrida \\
\hline ROK & SDI/ Bayswater, Victoria, Austrália & Híbrida \\
\hline Durafill & Kulzer/ Hanau, Alemanha & Microparticulada \\
\hline Ultrafill & Biodinâmica/ Ibiporã, Paraná, Brasil & Microhíbrida \\
\hline
\end{tabular}

Os corpos de prova foram confeccionados com auxílio de uma matriz de aço (TDV, Pomerode - SC, Brasil) rebitada, contendo um orifício de $30 \mathrm{~mm}$ de diâmetro por $5 \mathrm{~mm}$ de altura, replicando a metodologia do estudo de Migot et al. ${ }^{10}$, de forma adaptada, a fim de evitar sombreamento, ou qualquer interferência da matriz de aço no núcleo do corpo de prova, garantindo assim uma maior confiabilidade e fidelidade de cor durante a análise. Para auxiliar 0 manuseio dos espécimes, foi utilizado um fio ortodôntico 0,30 mm (Morelli, Sorocaba, SP, Brasil) para a criação de uma alça para fechamento do anel e para favorecer a movimentação e o transporte do corpo de prova (Figura 2).

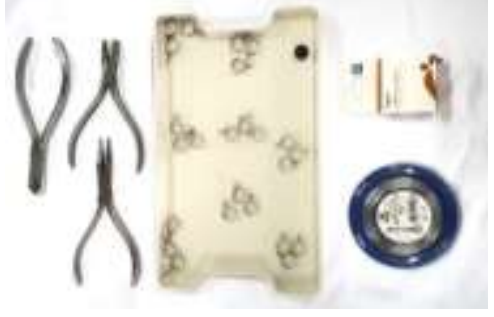


Para inserção da resina no molde, foi utilizada uma placa de vidro grossa (Daufenbach, Criciúma, SC, Brasil) para apoiar e, a seguir, foi feita a inserção dos incrementos de resina composta seguindo o tamanho recomendado pelos fabricantes de cada marca, utilizando-se a técnica incremental. Os compósitos foram pressionados no anel com auxílio de uma tira de poliéster (Império Dental, Jaboatão dos Guararapes - PE, Brasil) e uma lamínula de vidro 0.13 a $0.16 \mathrm{~mm}$ (Precision Glass Line, China), para permitir o escoamento do excesso de material, sendo feita a limpeza da placa a cada confecção e descarte da lamínula e tira de poliéster utilizadas (Figura 3).

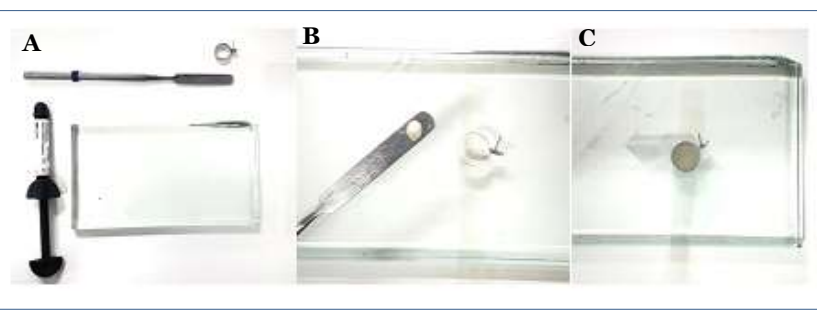

Figura 3 (A, B e C): Inserção da resina composta na matriz de aço (Fonte: Dados da pesquisa, 2020).

Logo após a inserção dos incrementos e retirada do excesso de material com a lamínula de vidro, foi realizada a fotoativação sob a matriz de poliéster e lamínula de vidro durante o tempo recomendado por cada fabricante, utilizando um fotopolimerizador de LED (SAEVO, Ribeirão Preto - SP, Brasil) com comprimento de onda compreendido entre $420 \mathrm{~nm}$ a $500 \mathrm{~nm}$ (luz azul), a qual possui intensidade ideal para integrar-se com a canforoquinona, de potência máxima de $6 \mathrm{~W}$, potência da luz: $1200 \mathrm{~mW} / \mathrm{cm} 2 \pm 200 \mathrm{~mW} / \mathrm{cm} 2$, semicondutor LED (InGaN) e fibra óptica $100 \%$ coerente, que garante a passagem de luz sem perdas (ø8mm 60ํㅡㄹ curva) (Figura 4).

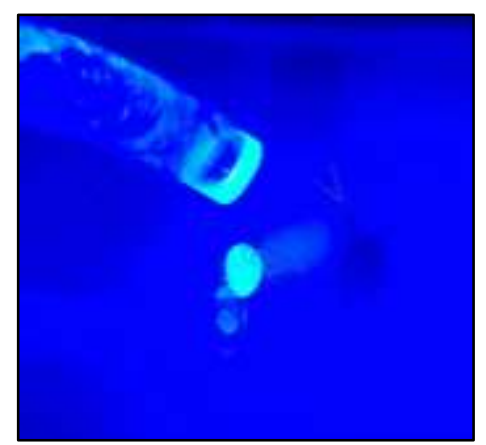

Figura 4: Polimerização dos corpos de prova de resina composta (Fonte: Dados da pesquisa, 2020).

Os corpos de prova foram fotopolimerizados e armazenados em recipiente plástico com tampa por um período de 7 dias. Após essa etapa, a cor obtida foi verificada por meio do escaneamento digital, realizado no centro de diagnóstico Medical Face. O equipamento utilizado foi o Scanner Intraoral
Trios3® (3Shape, Copenhagen, Dinamarca), previamente calibrado através do Kit de Calibragem para Cores do Trios $3 \AA$, e habilitado para tonalidades com o software 1.18.4.4 do TRIOS $3 \AA$, para a moldagem digital dos corpos de prova, um a um (Figura 5).

O scanner fotografa em alta qualidade, escaneia, ilumina, grava e mede automaticamente a tonalidade dos campos de prova. As imagens obtidas podem ser ampliadas em até 60 vezes e não apresentam ruídos, nem distorções. Para evitar interferência do sombreamento da matriz metálica e discordância comparativa de um corpo para outro, a seleção de cor dos corpos foi feita através do núcleo de cada corpo de prova.

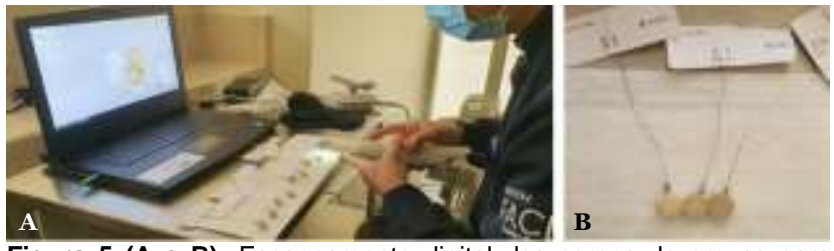

Figura 5 (A e B): Escaneamento digital dos corpos de provas para obtenção da cor (Fonte: Dados da pesquisa, 2020).

As imagens capturadas foram direcionadas para o software supracitado, auxiliando na construção de uma moldagem digital do corpo de prova (Figura 6). Através da moldagem obtida, a seleção de cor foi feita através de todos os valores de cor obtidos dos corpos de prova, comparado com os valores pré-estabelecidos da Escala Vita 3D Master, do Trios $3 \AA$.

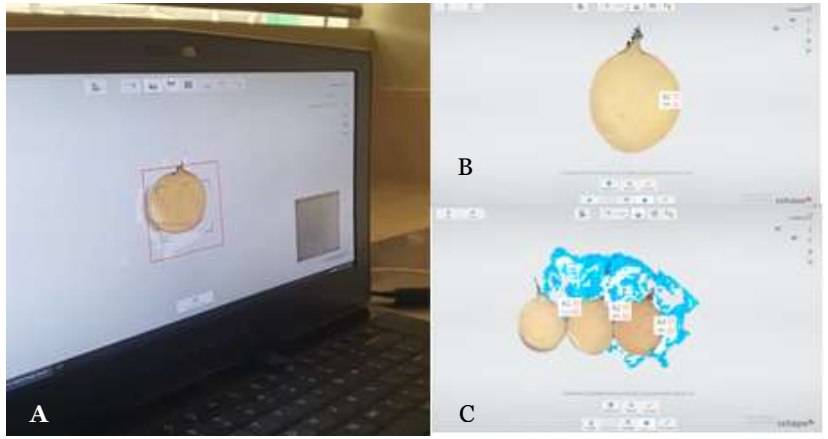

Figura 6 (A, B e C) - Escaneamento digital dos corpos de provas para obtenção da cor (Fonte: Dados da pesquisa, 2020).

Os mesmos corpos de provas foram submetidos, em outro ambiente do centro diagnóstico, à análise visual da cor, com auxílio da escala Vita Classical. Segundo Baratieri ${ }^{11}$ (2008), existem diferentes tipos de escala de cor, porém a maioria segue 0 padrão estabelecido pela Vita Classical, produzida pela companhia Vita.

Posteriormente, realizou-se a limpeza do corpo de prova para estimativa do brilho aparente e do matiz dominante, e selecionada a cor adequada mais semelhante no guia de tonalidade. Os corpos de prova e o dente tonalidade guia foram umedecidos. $O$ guia de 
tonalidade foi mantido perto do corpo de prova, para ser comparado com a cor adequada, sendo a observação feita de forma lateral, para diferenciar melhor a estimativa de brilho, analisando as diferenças de matiz e da saturação.

Foi desconsiderado o efeito total com os lábios relaxados, por não se tratar de um paciente em si. A verificação não passou de 5 segundos no corpo de prova para evitar a adaptação do matiz, sendo feita a observação de um papel azul entre cada período de verificação, pois é recomendado observar uma cor fria, como por exemplo azul, para não induzir o erro de semelhança ao avaliar uma cor quente novamente, como no caso de branco, amarelo e marrom.

O avaliador que utilizou o Scanner foi um profissional credenciado do laboratório, com experiência clínica. E a avaliação do corpo de prova, visualmente, foi realizado por um único pesquisador, 0 qual não conhecia os espécimes.

Foram usadas várias fontes de luz: a luz do dia para a correção da cor, depois lâmpada fluorescente, lâmpada incandescente e verificado o metamerismo (efeito que ocorre quando se observa cores semelhantes em condições de luminosidade distintas), sendo selecionada uma lâmpada semelhante a luz do dia, com 5000 a 6500 kelvin, considerada padrão para tomada de cor e por ser uma luz mais presente no dia a dia dos pacientes.

Foi escolhido o matiz mais exato ou mais próximo com brilho mais alto e saturação mais baixa; a modificação das faixas de cor com as próprias tintas não foi necessário e assim selecionada a cor mais aproximada.

Realizou-se a estatística descritiva para caracterização da amostra, por meio de números absolutos e percentuais. O Teste Quiquadrado e o Teste de Fisher foram utilizados para comparar as frequências das coincidências pelo método visual e digital, de acordo com a marca, cor e método. As análises foram realizadas no Software Statistical Package for the Social Sciences (SPSS), versão 20, com nível de significância de 5\%.

RESULTADOS

Em relação a tomada de cor, com auxílio do scanner digital, apenas $7(29,17 \%)$ resinas apresentaram correspondência entre a cor prometida pelo fabricante e a efetivamente constada com o equipamento. As resinas Ultrafill (Biodinâmica) e ROK (SDI) não apresentaram nenhuma correspondência com o proposto, sendo identificado uma única cor para todos os 6 diferentes tipos, a B2, como se não houvesse nenhuma diferenciação entre as 3 cores que o material deveria apresentar após a polimerização (Tabela 1).

A resina Charisma (Kulzer) foi a que apresentou maior correspondência, sendo as cores A1 e A3 avaliadas de forma concordante. Enquanto as resinas Durafill (Kulzer), Spectra Smart (Denstply), Fill Magic (Coltene), Z100 (3M) e Liss (FGM) apresentaram cores divergentes do proposto em duas das três resinas avaliadas de cada marca.

Tabela 1. Tomada de cor obtida com o scanner digital dos diferentes tipos de resina após polimerização.

\begin{tabular}{|c|c|c|c|}
\hline \multirow{3}{*}{ RESINAS - MARCAS } & \multicolumn{3}{|c|}{ CORES PROMETIDAS } \\
\hline & A1 & A2 & $\mathbf{A 3}$ \\
\hline & \multicolumn{3}{|c|}{ CORES OBTIDAS } \\
\hline KULZER / CHARISMA & A1 & & \\
\hline KULZER / CHARISMA & & B2 & \\
\hline KULZER / CHARISMA & & & $\mathrm{A} 3$ \\
\hline BIODINÂMICA / ULTRAFILL & B2 & & \\
\hline BIODINÂMICA / ULTRAFILL & & B2 & \\
\hline BIODINÂMICA / ULTRAFILL & & & B2 \\
\hline SDI / ROK & B2 & & \\
\hline SDI / ROK & & B2 & \\
\hline SDI / ROK & & & B2 \\
\hline KULZER / DURAFILL & B2 & & \\
\hline KULZER / DURAFILL & & B2 & \\
\hline KULZER / DURAFILL & & & $\mathrm{A} 3$ \\
\hline DENTSPLY / SPECTRA SMART & B2 & & \\
\hline DENTSPLY / SPECTRA SMART & & A2 & \\
\hline DENTSPLY / SPECTRA SMART & & & A2 \\
\hline COLTENE / FILL MAGIC & A1 & & \\
\hline COLTENE / FILL MAGIC & & B2 & \\
\hline COLTENE / FILL MAGIC & & & A2 \\
\hline $3 \mathrm{M} / \mathrm{Z100}$ & B1 & & \\
\hline $3 \mathrm{M} / \mathrm{Z100}$ & & $\mathrm{C} 1$ & \\
\hline $3 \mathrm{M} / \mathrm{Z100}$ & & & $\mathrm{A}_{3}$ \\
\hline FGM / LISS & B2 & & \\
\hline FGM / LISS & & B2 & \\
\hline FGM / LISS & & & $\mathrm{A}_{3}$ \\
\hline
\end{tabular}

correspondência da cor em relação às marcas das resinas, obteve-se $p=0,704$ no teste do Quiquadrado, havendo diferenças significativas $(p>0,05)$ entre os diferentes produtos testados. Quando considerada a cor do produto testado (A1, A2 e A3), também foram observadas diferenças significativas nas correspondências obtidas após polimerização, na análise pelo método digital $(p=0,244)$.

Apenas $10(41,67 \%)$ resinas tiveram correspondência de cor quando avaliadas pelo método visual. Todas as resinas das marcas ROK (SDI) e Durafill (Kulzer) apresentaram divergências quando avaliadas, merecendo destaque a ROK, cujas cores avaliadas distorceram para a faixa $B$, considerada mais escura que a proposta pelo produto. As resinas da 3M, FGM e Biodinâmica, obtiveram apenas uma unidade com correspondência de cor (Tabela 2).

Duas amostras da resina 3M/Z100, a A1 e A2, apresentaram uma grande distorção, pois foram avaliadas como $\mathrm{C} 1$ e $\mathrm{C} 2$ pelo método visual, consideradas as faixas de cores mais escuras da escala. A resina Charisma (Kulzer), assim como no método digital, foi a que 
apresentou maior correspondência de cores, sendo $100 \%$ coerente a avaliação visual com o proposto pelo fabricante.

Assim como na análise digital, as resinas ROK (SDI), apresentaram total distorção de cor, quando avaliadas pelo método visual, tendo apresentado cor B2 em todas as unidades avaliadas em ambos os métodos. A Durafill (Kulzer) também não apresentou nenhuma correspondência no método visual, o que também não foi coerente quando avaliado com o scanner. Tanto a Liss (FGM) quanto a Z100 $(3 \mathrm{M})$, também não conseguiram mais que um tipo com correspondência.

$\mathrm{Na}$ análise estatística da correspondência da cor pelo método visual clássico, também foram observadas diferenças significativas quando consideradas as marcas $(p=0,173)$, entretanto estas foram menores do que na avaliação via scanner digital. Com relação à cor proposta pelo fabricante $(\mathrm{A} 1, \mathrm{~A} 2 \mathrm{e}$ A3), também houve diferença relevante, sendo maior que os dados da análise digital $(p=0,842)$.

Quando submetidos à análise estatística para comparação do nível de correspondência das cores obtidas após a polimerização, constatou-se que as cores propostas não foram identificadas nem pelo método digital e nem pelo método visual $(92,6 \%)$, com diferença significativa. Ao comparar as frequências de coincidências, houve mais coincidências pela identificação da cor pelo método visual do que pelo método digital $(p=0,009$ pelo Teste de Fisher).

Tabela 2. Tomada de cor obtida pelo método visual com auxílio da Escala Vita Classical

\begin{tabular}{|c|c|c|c|}
\hline \multirow{3}{*}{ RESINAS - MARCAS } & \multicolumn{3}{|c|}{ CORES PROMETIDAS } \\
\hline & A1 & A2 & A3 \\
\hline & \multicolumn{3}{|c|}{ CORES OBTIDAS } \\
\hline KULZER / CHARISMA & A1 & & \\
\hline KULZER / CHARISMA & & A2 & \\
\hline KULZER / CHARISMA & & & $\mathrm{A} 3$ \\
\hline BIODINÂMICA / ULTRAFILL & A1 & & \\
\hline BIODINÂMICA / ULTRAFILL & & B1 & \\
\hline BIODINÂMICA / ULTRAFILL & & & B2 \\
\hline SDI / ROK & B2 & & \\
\hline SDI / ROK & & B2 & \\
\hline SDI / ROK & & & B2 \\
\hline KULZER / DURAFILL & A2 & & \\
\hline KULZER / DURAFILL & & A1 & \\
\hline KULZER / DURAFILL & & & A2 \\
\hline DENTSPLY / SPECTRA SMART & A1 & & \\
\hline DENTSPLY / SPECTRA SMART & & A2 & \\
\hline DENTSPLY / SPECTRA SMART & & & $\mathrm{A} 2$ \\
\hline COLTENE / FILL MAGIC & A1 & & \\
\hline COLTENE / FILL MAGIC & & A2 & \\
\hline COLTENE / FILL MAGIC & & & $\mathrm{A} 2$ \\
\hline $3 \mathrm{M} / \mathrm{Z100}$ & $\mathrm{C} 1$ & & \\
\hline $3 \mathrm{M} / \mathrm{Z} 100$ & & $\mathrm{C} 2$ & \\
\hline $3 \mathrm{M} / \mathrm{Z1OO}$ & & & $\mathrm{A} 3$ \\
\hline FGM / LISS & B2 & & \\
\hline FGM / LISS & & B2 & \\
\hline FGM / LISS & & & $\mathrm{A} 3$ \\
\hline Fonte: Dados da pesquisa, 2020 & & & \\
\hline
\end{tabular}

A exigência estética tem crescido consideravelmente entre a população mundial, contribuindo para que os tratamentos restauradores estéticos sejam os mais requeridos pelos pacientes da clínica odontológica. Essa realidade resulta no aumento da necessidade constante de treinamento por parte dos cirurgiões dentistas que buscam fornecer resultados de excelência em seus procedimentos ${ }^{12,13}$.

O sucesso ou o fracasso das restaurações em resina composta está relacionada a escolha do material e de suas características físicas e químicas. Entre os principais fatores que influenciam na construção de uma restauração estética satisfatória estão as propriedades ópticas que são classificadas com base em dimensões, como a matiz, croma, valor, opalescência, fluorescência, entre outros. Assim, há de se considerar que, buscando-se alcançar uma restauração estética de qualidade, a etapa de seleção de cor é considerada crítica e de extrema importância, evidenciando-se como um grande desafio $5,6,12,14$.

Baseado na relevância da temática, este estudo buscou verificar a correspondência de cor das resinas compostas em relação à Escala Vitta, através do método visual e pelo método digital com o auxílio de um scanner digital, com a finalidade de auxiliar aos profissionais da Odontologia na escolha dos métodos de verificação da cor, e dos materiais que melhor reproduzam a estética dental.

No presente estudo, ao ser avaliado a cor das resinas compostas através do espectrofotômetro, apenas $29,17 \%$ das amostras apresentaram correspondência entre a cor informada na embalagem e a constatada pelo equipamento, sendo encontrada diferenças estatísticas significativas. Esse resultado corrobora com os achados de Alves et al. ${ }^{6}$, onde - espectrofotômetro VITA Easyshade $\AA$ Compact fez leituras de cor distintas entre a escala Vita Classical e as resinas compostas avaliadas. Entretanto, ao analisar os resultados encontrados por Dantas et al. ${ }^{15}$, os achados dessa pesquisa foram superiores, já que no estudo supracitado nenhuma das resinas compostas apresentou correspondência de cor com a referência na escala Vita.

Neste contexto, Migot et al. ${ }^{10}$ buscaram avaliar a cor de resinas compostas de diferentes marcas comerciais utilizando o espectrofotômetro VITA Easyshade ${ }^{\circledR}$ Compact. Os resultados evidenciaram que a cor A2 de todas as resinas compostas e a cor A3 da maior parte das resinas analisadas apresentaram diferenças estatísticas em relação a escala utilizada. Dessa forma, constataram que a maioria das resinas estudadas não apresentou correspondência de cor com a escala Vita.

$\mathrm{Na}$ maioria dos casos, a cor obtida pela avaliação do scanner foi mais escura, segundo 
a escala do que o proposto, obtendo tonalidades na faixa $B$ e até mesmo na $C$, em apenas um caso (Z100 - 3M, na cor proposta A2). Resultados semelhantes foram encontrados por Dantas et al. ${ }^{15}$ (2011), onde todos os corpos de prova da cor A1 e A2 foram classificados como $\mathrm{C} 3$, B3 e B4, tons mais escuros da escala. Uma das possíveis hipóteses para essa mudança na identificação da cor pode ser a translucidez apresentada pelas resinas compostas, que deixam atravessar parte da luz incidente e acarretam uma mudança na leitura do espectrofotômetro, já que esses equipamentos analisam a luz que retorna do objeto ${ }^{15}$.

Ao realizar a análise estatística desse estudo, notou-se uma diferença estatística entre cor das diversas marcas de produtos avaliados. Nesta perspectiva, Dantas et al. ${ }^{15}$ e Migot et al. ${ }^{10}$ concluíram que a maioria das resinas compostas testadas apresentaram tonalidades semelhantes, mas estatisticamente diferentes entre si. Tendo em vista essas discrepâncias de cor entre as marcas, tem-se considerado inviável a substituição na clínica de uma resina por outra de marca comercial diferente.

Realizando uma avaliação da cor das resinas compostas através do método visual, apenas $41,67 \%$ das amostras apresentaram correspondência de cor, sendo uma diferença estatística considerável. Esse dado está em consonância com os achados de Vieira et al. ${ }^{16}$, onde concluiu-se que existe divergência entre a cor da resina composta avaliadas pelo método visual e a escala Vita Classical. Já no estudo realizado por Dantas et al. ${ }^{15}$, os resultados mostraram que, na avaliação pelo método visual, observou-se baixa correspondência de cor das resinas avaliadas tanto em relação à escala Vita como entre si, evidenciando a alta subjetividade e baixa reprodutibilidade dessa comparação.

O método visual de avaliação de cor é um procedimento de interpretação individual, dependendo da interação de diferentes fatores, o que torna a equiparação e a padronização da comunicação das cores difíceis. Apesar de ser considerado um método subjetivo, a análise visual da cor ainda é o método de seleção mais utilizado na Odontologia. Quando usada, devese levar em consideração a fonte de luz do consultório, fadiga visual, desidratação do dente, idade, experiência e maior sensibilidade ocular $^{5,17,18}$.

Com objetivo de minimizar as discrepâncias na identificação visual da cor, foram introduzidos no mercado instrumentos eletrônicos para facilitar a seleção. Estes têm por finalidade reduzir ou superar imperfeições e incoerências da escolha de cor, pelo método visual tradicional ${ }^{19}$. Entretanto, ainda há muitas controvérsias e divergências em relação a eficiência dos dois métodos, tanto na avaliação da cor da estrutura dentária quanto na cor das resinas compostas.

Buscando compreender e comparar a eficácia dos métodos digital e visual, realizou-se a análise estatística dos resultados, sendo observado que nenhum dos dois métodos identificou adequadamente as cores das resinas compostas. Entretanto, as cores coincidentes foram mais identificadas pelo método visual do que digital. Estes achados corroboram o estudo de Parameswaran et al. $^{20}$, onde os resultados mostram que o método visual foi mais preciso que 0 método espectrofotométrico. 0 espectrofotômetro mostrou melhores índices de concordância entre os avaliadores, independentemente do guia de cores utilizado. Enquanto o guia de cores VITAPAN Classical foi mais preciso com o espectrofotômetro, o guia de cores VITAPAN 3D Master mostrou-se melhor com o método visual. Em contrapartida, Liberato $^{17}$ encontrou em seu estudo que os métodos instrumentais são mais confiáveis e reprodutíveis que o método visual.

Segundo Dantas et al. ${ }^{15}$, o método visual mostrou ser bastante preciso quando a diferença entre os tons das resinas compostas é significativa, como entre A2 e A4, sendo notória a dificuldade de identificar cores muito próximas. Entretanto, o método digital apresentou-se mais eficiente em identificar a diferença entre as cores claras e escuras, como entre A1, A2 e A4, mesmo não apresentando correspondência com a escala Vita.

A partir dos resultados observados, torna-se evidente a importância dos cirurgiõesdentistas compreenderem as características ópticas das resinas compostas para alcançar um resultado estético satisfatório. Dessa forma, a realização de pesquisas que busquem esclarecer essas propriedades é primordial para o aperfeiçoamento das técnicas e dos procedimentos restauradores.

O presente estudo verificou a correspondência de cor das resinas compostas em relação à Escala Vita, através da técnica visual e com auxílio de um scanner digital, com a finalidade de auxiliar aos profissionais de Odontologia na escolha dos métodos de verificação da cor, e dos materiais que melhor reproduzam a estética dental. Apesar dos resultados satisfatórios, recomenda-se a realização de estudos clínicos randomizados utilizando metodologia semelhante ao presente 
estudo, comparando a eficácia da seleção de cor com outros equipamentos.

CONCLUSÃO

Diante do exposto, é possível concluir que em ambos os métodos, digital e visual clássico, houve pouca correspondência entre a cor proposta pelos fabricantes e a obtida após a polimerização das resinas e que, quando comparadas às frequências de coincidências, estas foram mais identificadas pela constatação da cor pelo método visual do que pelo método digital.

\section{REFERÊNCIAS}

1. Dias HB, Carrera ET, de Souza Rastelli AN. The influence of $\mathrm{pH}$ and chemical composition of beverages on color stability of a nanofilled composite resin. Gen Dent. 2016;64(6):21-7.

2. Pereira ASR, Pires MM, Figueiredo JAP, Mota EG, Pires LAG. Análise dos níveis de cinza de 4 resinas compostas micro híbridas utilizando um sistema de radiografia digital direto. Rev odonto ciênc. 2005;20(47):3-10.

3. Gómez-Polo C, Gómez-Polo M, CeleminViñuela A, Martínez Vázquez De Parga JA. Differences between the human eye and the spectrophotometer in the shade matching of tooth colour. J Dent. 2014;42(6):742-45.

4. Baharin S, Dong T, Jing T. Anterior Tooth Shade Selection Procedure: Influence of Light Sources and Patient's Position. Sains Malay. 2013; 42(1):7-11.

5. Pereira GN. Métodos de seleção de cor [monografia]. Belo Horizonte: Universidade Federal de Minas Gerais - UFMG; 2019.

6. Alves GKJ, Aued N, Soares FZM, Jacques LB, Kaizer MR, Mallmann A. Avaliação da cor de um compósito com espectrofotômetro em diferentes modos de leitura e condições de armazenagem. RFO UPF. 2014;19(1):101-6.

7. Reyes J, Acosta P, Ventura D. Repeatability of the human eye compared to an intraoral scanner in dental shade matching. Heliyon. 2019; 23(7):1-6.

8. Schmeling M., Maia $H$, Baratieri L. Opalescence of bleached teeth. J Dent. 2012; 40(1):35-9.

9. Hassel AJ, Cevirgen E, Balke Z, Rammelsberg $P$. Intraexaminer reliability of measurement of tooth color by spectrophotometry. Quintessence Int. 2009;40(5):421-26.

10. Migot I, Marques AB, Rocha I, Cavalcanti A, Argolo S. Avaliação de cor de diferentes marcas e sistemas de resina composta por espectrofotometria. Rev Bahiana Odontol. 2017;8(3):68-75.

11. Baratieri LN. Soluções clínicas - fundamentos e técnicas. Florianópolis: Editora Ponto; 2008.

12. Aguiar MM. A importância da escolha da cor para a realização de restaurações estéticas: revisão da literatura ilustrada [monografia]. Aracajú: Universidade Tiradentes; 2012.

13.Zavanelli AC, Sônego MV, Zavanelli RA, Mazaro JVQ, Falcón-Antenucci RM. Perception and expectation. What do patients really want from the dental treatment? RGO, Rev Gaúch Odontol. 2017;65(3):243-48.

14. Silva JC, Silva DR, Barbosa DN. Estabilidade de cor das resinas compostas: um desafio para a dentística restauradora. Arch Health Invest. 2017;6(10):451-57.

15. Dantas RAA, Florez FLE, Campos EA, Andrade MF, Saade JRC, Oliveira Júnior OB. Correspondência de cor de diferentes marcas e sistemas de resina composta em relação à escala vita classical. RPG. 2011;18(1):45-51.

16. Vieira APSB, Santos TKGL, Carvalho LGA, Patrício CEG, Galvão AKC, Silveira BC. Diferença de cor entre resinas compostas de lotes diferentes de acordo com a escala vita. Rev Campo do Saber. 2018;4(5):86-100.

17. Liberato WF. Comparação de correspondência de cores in vivo entre métodos visuais, escaner intraoral e com espectofotômetro [dissertação]. Niterói: Universidade Federal Fluminense;2018.

18. Corcodel N, Rammelsberg $\mathrm{P}$, Jakstat $\mathrm{H}$, Moldovan O, Schwarz S, Hassel AJ. The linear shade guide design of Vita 3D-master performs as well as the original design of the Vita 3Dmaster. J Oral Rehabil. 2010;37(11):860-65.

19. Lasserre JF, Pop-Ciutrila IS, Colosi HA. A comparison between a new visual method of colour matching by intraoral camera and conventional visual and spectrometric methods. J Dent. 2011;39 (3):29-36.

20. Parameswaran V, Anilkumar S, Lylajam S, Rajesh C, Narayan V. Comparison of accuracies of an intraoral spectrophotometer and conventional visual method for shade matching using two shade guide systems. J Indian Prosthodont Soc. 2016;16(4):352-58.

\section{CONFLITO DE INTERESSES}

Os autores declaram não haver conflitos de interesse

AUTOR PARA CORRESPONDÊNCIA

\section{Kauana da Silva Andrade}

Centro Universitário de João Pessoa (UNIPÊ)

Rodovia BR-230, km 22, S/N - Água Fria

58053 - 000 João Pessoa - PB, Brasil.

E-mail: kauanaandrade12@gmail.com 